\title{
Mindfulness e meditação: Práticas complementares ao tratamento do paciente com DPOC
}

\author{
João G. Monteiro, ${ }^{1,2}$ Yves Raphael de Souza, ${ }^{1,2}$ Patricia X. H. Frasson, ${ }^{1,2}$ Diego Condesso, ${ }^{1}$ \\ Bianca Figueira, ${ }^{1}$ Kenia M. da Silva ${ }^{1,2 *}$
}

\begin{abstract}
Resumo
Introdução: No Brasil, calcula-se que 10 milhões de pessoas tenham doença pulmonar obstrutiva crônica (DPOC). A depressão e a ansiedade são distúrbios observáveis em mais de um terço dos pacientes com DPOC. A meditação e o mindfulness têm sido indicados para redução do estresse e melhora da qualidade de vida, sendo utilizados no abrandamento dos sintomas da DPOC. Objetivo: Analisar a inserção da meditação e do mindfulness como tratamento complementar para os pacientes com doença pulmonar obstrutiva crônica (DPOC). Método: Pesquisa descritiva, com síntese feita em forma de resumo textual das características e das informações relevantes das evidências científicas sobre a inserção de meditação e mindfulness na abordagem terapêutica dos pacientes com doenças pulmonar obstrutiva crônica. Os bancos de dados eletrônicos utilizados foram: Pubmed, Scielo, Bireme, Cochrane, Medline, PEDro. As palavras-chave são: DPOC, ansiedade, depressão, meditação, mindfulness. O período pesquisado foi de 11 anos (2005-2016). Resultados: Foram encontrados treze artigos, dos quais três foram artigos de revisão bibliográfica e dez artigos foram trabalhos experimentais. Conclusão: $\mathrm{O}$ uso das práticas de mindfulness e de meditação podem funcionar como instrumentos terapêuticos complementares importantes no abrandamento dos sintomas observados na DPOC, auxiliando na diminuição da sensação de dispneia, ansiedade, estresse e depressão. Apesar disso, foram publicados poucos trabalhos experimentais sobre o tema, evidenciando a necessidade de uma pesquisa mais aprofundada.
\end{abstract}

Descritores: Doença pulmonar obstrutiva crônica (DPOC); Ansiedade; Depressão; Meditação; Mindfulness.

\begin{abstract}
Mindfulness and meditation: Complementary practices in the treatment of patients with chronic obstructive pulmonary disease

Introduction: In Brazil, an estimated 10 million people have chronic obstructive pulmonary disease (COPD). Depression and anxiety are present in over a third of COPD patients. Meditation and mindfulness are techniques indicated for stress reduction and promoting better quality of life, as well as in situations for softening the symptoms of COPD.
\end{abstract}

1. Universidade Veiga de Almeida, Faculdade de Fisioterapia, Rio de Janeiro, RJ, Brasil.

2. Universidade do Estado do Rio de Janeiro. Departamento de Pneumologia, Rio de Janeiro, RJ, Brasil.

*Endereço para correspondência:

Estrada dos Bandeirantes, 7777, bloco 1, ap. 606

Rio de Janeiro, RJ, Brasil.

CEP: 22783-115

E-mail: kenia.msilva@gmail.com

BJHBS, Rio de Janeiro, 2019;18(1):41-46

Recebido em 20/09/2017. Aprovado em 05/02/2018.

Objective: To analyse the use of meditation and mindfulness as a complementary treatment for patients with chronic obstructive pulmonary diseases. Method: Descriptive trial. The synthesis was done in the form of a textual summary of the characteristics and relevant information of the scientific evidence on the use of meditation and mindfulness in the therapeutic approach for patients with chronic obstructive pulmonary diseases over 11 years, from 2005 to 2016. The electronic databases Pubmed, Scielo, Bireme, Xochrane, Medline and PEDro were used. The keywords were COPD, anxiety, depression, meditation and mindfulness. Results: Three review and ten intervention trial articles were found, comprising a total of 13. Conclusion: The use of mindfulness and meditation practices may be important therapeutic tools to treat the symptoms observed in COPD. They may be a valuable adjunct to pulmonary rehabilitation and traditional drug therapy, since they help in the sensation of dyspnea, producing a decrease in anxiety, stress and depression observed as co-morbidity of this disease. However, few experimental papers about meditation and mindfulness have been published. More studies about this type of intervention should be conducted.

Keywords: Chronic obstructive pulmonary disease (COPD); Anxiety; Depression; Meditation; Mindfulness. 


\section{Resumen}

Mindfulness y meditación: Práctica complementaria al tratamiento del paciente con enfermedad pulmonar obstructiva crónica

Introdución: En Brasil, se estima que 10 millones de personas tenen EPOC. La depresión y la ansiedad son trastornos observables en más de un tercio de los pacientes con EPOC. La meditación y la atención plena han sido indicadas para reducir estrés y mejor calidad de vida, recomendadas para aliviar los síntomas de EPOC. Objetivo: Analizar la inserción de la meditación y mindfulness como tratamiento complementario para los pacientes con enfermedad pulmonar obstructiva crónica (EPOC). Método: Investigación descriptiva, siendo que la síntesis se hará en forma de resumen textual de las características y de las informaciones relevantes de las evidencias científicas sobre la inserción de la meditación y mindfulness en el abordaje terapéutico de los pacientes con enfermedades pulmonar obstructiva

\section{Introdução}

Atualmente a Organização Mundial de Saúde (OMS) estima que 65 milhões de pessoas no mundo tenham o diagnóstico de doença pulmonar obstrutiva crônica (DPOC), classificada de moderado a grave, com um prognóstico de que, até 2030, essa seja a terceira causa de morte no mundo. ${ }^{1}$ No Brasil, calcula-se que 10 milhões de pessoas tenham DPOC, ${ }^{2}$ com maior incidência em homens com mais de 75 anos. ${ }^{3}$

As diretrizes da Iniciativa Global para DPOC, o Gold Obstructive Lung Disease (GOLD), classificam a gravidade da doença com base na VEF1 e na frequência de exacerbações, e definem a DPOC como uma doença comum prevenível e tratável, caracterizada pela limitação persistente de fluxo aéreo não totalmente reversível, normalmente progressiva, associada a uma resposta inflamatória crônica aumentada nos brônquios e nos pulmões, a partículas ou gases tóxicos, excluindo a presença de outras doenças que causam obstrução do fluxo aéreo. ${ }^{4,5} \mathrm{~A}$ DPOC tem sido associada, em alta prevalência, com outras comorbidades, que aumentam em número e gravidade com a idade, e se apresentam em maior número em grupos sociais de baixa renda.[6] A depressão e a ansiedade são distúrbios frequentemente observáveis, apresentados por mais de um terço dos pacientes com DPOC, que unidos a outros sintomas podem também contribuir para a progressão da doença.7.8 cronica. Los bancos de datos electrónicos utilizados fueron: Pubmed, Scielo, Bireme, Cochrane, Medline, PEdro. Las palabras clave fueron: EPOC, ansiedad, depresión, meditación, mindfulness. En un espacio de tiempo delimitado de 11 años (2005 - 2016). Resultados: Se han encontrado trece artículos, de los cuales tres han sido artículos de revisión bibliográfica y diez artículos con trabajos experimentales. Conclusión: El uso de las prácticas de mindfulnes y meditación puede funcionar como importantes instrumentos terapéuticos en la ralentización de los síntomas observados en el EPOC. Puede ser un complemento valioso a la rehabilitación pulmonar y terapia medicamentosa tradicional ya que auxilia en la disminución de la sensación de disnea, así como en la disminución de la ansiedad, el estrés y la depresión. A pesar de ello, pocos trabajos experimentales sobre el tema fueron publicados, dejando claro la necesidad de una investigación más profundizada sobre el tema.

Palabras clave: Enfermedad pulmonar obstructiva crónica (EPOC); Ansiedad; Depresión; Meditación; Mindfulness.
A depressão é definida como um problema comum de saúde mental que vem acompanhado por um alto grau de aflição emocional e prejuízo funcional, incluindo diminuição do humor e perda de interesse ou prazer em atividades da vida diária (AVDs), tornando o paciente estigmatizado e isolado. Outros sintomas incluem fadiga ou sensação de falta de energia, mudanças significativas no peso, apetite e sono, sensação de culpa, baixa autoestima, falta de concentração, pessimismo e tendência suicida. Evidências sugerem que pacientes com depressão têm expectativa de vida reduzida entre 5 e 10 anos em relação a pacientes sem essas desordens, e que eles morrem pela combinação da depressão com desordens clínicas, como doença vascular, diabetes, DPOC, asma e câncer. ${ }^{9}$

Ansiedade é também um problema comum de saúde mental que é associado a um sentimento vago e desagradável de medo, apreensão, caracterizado por tensão ou desconforto, derivado de antecipação de perigo, de algo desconhecido ou estranho. Ansiedade e medo passam a ser considerados patológicos quando exagerados, ou desproporcionais ao estímulo, e também quando interferem na qualidade de vida, conforto emocional e desempenho diário do indivíduo. ${ }^{10}$ Os sintomas físicos se resumem em cansaço, fadiga, tensão muscular, cefaleias, dificuldade para adormecer ou 
sono insatisfatório, boca seca, mãos ou pés úmidos, enjoos ou diarreia, aumento da frequência urinaria, sudorese excessiva, tremores, coração acelerado, tonturas, desconforto abdominal, respiração acelerada, palpitações (sensação de que o coração falhou uma batida) e micção frequente.11 Para pessoas com DPOC, a ansiedade pode levar à hiperventilação, à sensação de falta de ar, o que provoca aumento da ansiedade. ${ }^{12}$ A dispneia pode ser fortemente influenciada por ansiedade, questões afetivas e estresse.

Tanto a ansiedade quanto a depressão precisam ser controladas para a melhoria da qualidade de vida do paciente, sendo indicado o tratamento farmacológico. A ansiedade leva o paciente a uma inatividade cada vez maior, devido ao receio de uma possível crise de dispneia. ${ }^{13}$ Pode inclusive contribuir para uma hipersensibilidade à dispneia ou uma percepção exagerada ou distorcida da gravidade da disfunção pulmonar. $^{14}$

As técnicas de relaxamento têm sido utilizadas no abrandamento dos sintomas da DPOC.O uso regular das técnicas, nos períodos de dispneia, se propõe a reduzir os níveis de ansiedade, estresse e temor pela possibilidade das crises. Segundo Hyland e colaboradores, ${ }^{15}$ há uma evidência favorável no uso dessas técnicas.

A meditação e o mindfulness têm sido indicados para redução do estresse e melhora da qualidade de vida. Segundo Bianco e colaboradores, ${ }^{16}$ a meditação tem sido descrita como um foco mental em objetos, em si mesmo, em sensações físicas, emoções e pensamentos. Pode ter abordagem religiosa, espiritual e filosófica ou pode ser utilizada para melhora das condições emocionais. Os mesmos autores descrevem mind fulness como similar à meditação, porém com propósitos diferentes. A meditação objetiva a autocompreensão e a diminuição das aflições, enquanto o mindfulness está orientado para o bem-estar psicológico e tratamento de psicopatologias, como uma abordagem mais ocidental criada para integrar a prática clínica com as técnicas orientais. Muitos protocolos surgiram e são conhecidos como Intervenções Baseadas em Mindfullness (em inglês, Mindfullness-Based Interventions ou MBIs). Esses protocolos foram montados para auxiliar o praticante a observar o momento presente imediato, de forma atenta e focada. ${ }^{16}$

Tanto a meditação como o mindfulness podem também enfatizar a respiração como foco da meditação, trazendo experiências respiratórias positivas. Dessa forma, podem ser capazes de alterar parâmetros respiratórios, controlar os níveis de ansiedade e a intensidade de sintomas do DPOC ${ }^{12}$, e assim diminuirem a utilização de fármacos para esse fim.

O presente trabalho teve como objetivo de analisar, através de uma revisão descritiva da literatura, a meditação e o mindfulness como técnicas complementares ao tratamento da DPOC, no controle da depressão e da ansiedade, observáveis na maioria dos pacientes com DPOC.

\section{Metodologia}

O estudo é uma pesquisa descritiva, em forma de resumo textual, das características e informações relevantes das evidências científicas sobre a inserção da meditação e de mindfulness na abordagem terapêutica dos pacientes com doenças respiratórias crônicas. Foram utilizados os seguintes bancos de dados eletrônicos: Pubmed, Scielo, Bireme, Cochrane, Medline, PEDro. As palavras-chave utilizadas foram: DPOC, ansiedade, depressão, meditação, mindfulness. Os artigos foram delimitados a um período de 11 anos (2005-2016), o que resultou em 13 artigos sobre os temas propostos, publicados no período entre 2005 e 2016, dos quais 3 foram artigos de revisão e 10 foram artigos sobre pesquisas de intervenção.

\section{Resultados}

As revisões encontradas foram:

Farver-Vestergaard ${ }^{17}$ encontraram 20 estudos, que investigaram 1361 pacientes, e revelaram efeitos estatisticamente relevantes, com bom resultado nas intervenções psicossociais, sugerindo que essas técnicas podem ser uma ferramenta no tratamento do DPOC, na abordagem da depressão e da ansiedade. Panagioti e colaboradores ${ }^{7}$ encontraram 29 estudos experimentais com 2063 participantes, com abordagem em terapia cognitivo comportamental e relaxamento com base no mindfulness na reabilitação pulmonar, que demonstraram efeitos pequenos, mas significativos na redução da depressão e da ansiedade. A revisão realizada por Teneja ${ }^{18}$ observou a introdução da meditação, da yoga e da consciência corporal no contexto do tratamento com exercícios respiratórios em DPOC, em que foi encontrada melhora da capacidade funcional e da qualidade de vida, com diminuição do uso de medicamentos. Porém concluíram que essa terapêutica ainda precisa ser melhor observada, com demanda de quantidade de pesquisas.

Howarth e colaboradores ${ }^{19}$ realizaram um estudo experimental com objetivo de testar a eficiência de 


\section{Artigo de revisão}

uma abordagem com mindfulness em pacientes com doenças crônicas. Foram realizadas entrevistas em 18 pacientes portadores de doenças crônicas (DPOC, dor crônica e doença cardiovascular), buscando avaliar a aceitação e aplicabilidade dessa intervenção, com a participação de 14 pacientes. As entrevistas foram conduzidas após o uso de um áudio com base em mindfulness, em uma clínica e, uma semana após, o uso do áudio em sua própria residência. O estudo utilizou uma breve intervenção com mindfulness (10 minutos), para pacientes com doenças crônicas. Todos os pacientes relataram benefícios, como sensações de relaxamento e melhora no convívio com os sintomas, com boa aceitação do programa de tratamento.

Chan e colaboradores ${ }^{12}$ e Benzo ${ }^{20}$ realizaram estudo experimental randomizado de 8 semanas, com base na prática de MBSR (Mindfulness-Based Stress Reduction). Chan e colaboradores ${ }^{12}$ observaram que os pacientes que praticaram o MBSR apresentaram menos dispneia e ansiedade, se comparado com o grupo controle, que continuou com o programa regular de reabilitação pulmonar. Benzo ${ }^{20}$ propôs o programa de mindfulness. Através do uso dessa técnica, os autores chegaram à conclusão de que o efeito qualitativo da meditação como intervenção, se replicado em grandes grupos, pode revolucionar a forma como são ofertadas as oportunidades de tratamento e a comunicação com portadores de DPOC. Segundo os autores, essa intervenção também demonstrou ser um interessante modificador de padrões de vida de pacientes, representando a oportunidade de uma mudança positiva na vida dos participantes.

Mularski e colaboradores, ${ }^{21}$ diferentemente dos outros autores, não encontraram, nesse estudo, nenhuma melhora mensurável, sugerindo que essa intervenção não parece ser uma opção terapêutica para pacientes com DPOC de moderado a grave. Foi realizado um estudo experimental randomizado, de oito semanas, com o objetivo de testar a eficácia de uma terapia respiratória baseada em mindfulness (MBBT Mindfulness Based Breathing Therapy) para a melhora de sintomas e da qualidade de vida em indivíduos com DPOC. O grupo intervenção, com 19 indivíduos, foi submetido à prática de mindfulness e o grupo controle, com 25 indivíduos, foi submetido à terapia em grupo, para testar a melhora dos sintomas e de qualidade de vida de portadores de DPOC. Os resultados foram avaliados utilizando-se o teste de caminhada de 6 minutos (TC6), distância caminhada no teste de caminhada de 6 minutos (DTC6M), escala de dispneia de Borg, formulários de qualidade de vida, taxas de exacerbação e medidas de estresse e mindfulness.

No estudo de Harrison e colaboradores, ${ }^{22}$ indivíduos com DPOC se sentiram confortáveis utilizando a respiração para reduzir a ansiedade, mas sugeriram que são necessárias metodologias de avaliação para seus resultados. Os autores realizaram um estudo qualitativo, com 20 profissionais de saúde, com, pelo menos, 1 ano de experiência clínica em gestão de DPOCe 19 indivíduos com DPOC de moderado a grave. Propuseram, também, entrevistas semiestruturadas com o objetivo de explorar as opiniões dos profissionais de saúde e pacientes portadores de DPOC com relação à prática de mindfulness. A partir das entrevistas, concluíram que a meditação parece ser uma técnica terapêutica possível para indivíduos com DPOC, porém os profissionais de saúde e os pacientes devem ter compreensão e conhecimento da técnica.

Entrevistas e questionários também foram utilizados no estudo de Chan e Lehto ${ }^{23}$ para avaliar o resultado da intervenção de oito semanas, com uma hora de prática meditativa semanal para 41 pacientes, dos quais 32 pacientes contribuíram com experiências detalhadas sobre o aprendizado e a prática da meditação; 21 pacientes identificaram melhora dos sintomas físicos e emocionais, e 13 dos participantes ofereceram detalhes sobre como adaptaram as habilidades adquiridas na prática da meditação às suas vidas diárias, para melhorar a função emocional e diminuir a dispneia.

A prática da meditação também foi estudada inserida nas práticas corporais como a yoga e o tai chi chuan. Fulambarker e colaboradores ${ }^{24}$ realizaram um estudo que avaliou o efeito da yoga e da meditação em pacientes com DPOC, observando os efeitos na melhora da qualidade de vida e nos parâmetros de função pulmonar de 33 pacientes. Todos receberam tratamento padrão da doença. A prática foi realizada por 1 hora, 3 vezes por semana, durante 6 semanas, por um terapeuta de yoga certificado. A qualidade de vida e o teste de função pulmonar foram novamente verificados após as 6 semanas, em que foram observadas melhoras estatisticamente significativas. Os autores concluíram que a prática estudada resulta em melhora da qualidade de vida e da função pulmonar em curto prazo, porém, assim como Farver-Vestergaard ${ }^{17}$, Teneja $^{19}$, Harrison e colaboradores ${ }^{22}$ sugerem que pesquisa adicional é necessária para confirmar os resultados num estudo randomizado controlado e realizado por um período maior. 
Yeh e colaboradores ${ }^{25}$ realizaram um estudo com prática de meditação inserida no tai chi chuan, por uma hora, duas vezes por semana, em 12 semanas. Foram randomizados 10 pacientes com DPOC de moderado a grave. O grupo intervenção (5 pacientes) foi submetido à intervenção proposta e o grupo controle (5 pacientes) a cuidados usuais. Após 12 semanas, houve uma melhoria significativa na qualidade de vida, comparado com o grupo controle, porém não observaram melhora significativa no teste de caminhada em seis minutos. Assim como não houve mudanças significativas nos resultados de ambos os grupos para consumo máximo de oxigênio ( $\mathrm{VO}_{2} \mathrm{max}$ ). Os autores concluíram que a meditação e a prática do tai chi chuan em pacientes com DPOC de moderado a grave apresentaram resultados favoráveis, sugerindo que podem ser técnicas complementares ao tratamento, mas como os outros autores, acreditam que ainda necessitaria maior investigação.

Um estudo randomizado com grupo controle, duplo cego com 81 mineiros (36-60 anos), com estágios 2 e 3 de DPOC, foi realizado por Ranjita e colaboradores. ${ }^{26}$ Os autores estudaram o treinamento de yoga, aplicado por 90 minutos por dia, diariamente, por 12 semanas no grupo intervenção. O grupo controle recebeu tratamento usual. Após desistências, 36 pacientes restaram tanto no grupo intervenção, que praticaram a meditação e a yoga, como no grupo controle. Os resultados se mostraram significantes para diminuição da dispneia e da fadiga no grupo intervenção, assim como no teste de caminhada em seis minutos, na saturação periférica de oxigênio, na frequência cardíaca. Os autores concluíram que essa intervenção pode ser incluída como um tratamento adjunto à terapia convencional nos programas de reabilitação pulmonar para pacientes com DPOC.

Diferente dos outros autores, Selman e colaboradores ${ }^{27}$ utilizaram aulas televisionadas, às quais chamou de teleyoga, utilizando as técnicas de meditação, respiração e posturas, em um estudo controlado, não randomizado, por 8 semanas. Foram oferecidas a 15 pacientes portadores de insuficiência cardíaca e DPOC, sendo 7 para o grupo intervenção e 8 para o grupo controle. Para os pacientes que sofreram a intervenção, esta foi considerada aceitável e apropriada. Apesar de reportarem terem gostado da prática, eles relataram se sentirem isolados socialmente. Os autores concluíram que a teleyoga pode ser uma forma aceitável como tratamento complementar para pessoas com insuficiência cardíaca e DPOC. Os achados mostraram rumos interessantes para pesquisadores que utilizam práticas corporais, como a yoga, com intervenções televisionadas.

Quanto à prática meditativa e às técnicas de mindfulness, os autores encontram respostas positivas para a diminuição da ansiedade e da depressão. Quando associada a práticas corporais, como a yoga e o tai chi chuan, os autores observaram melhora na capacidade funcional, além da melhora na qualidade de vida dos pacientes com DPOC. Os estudos encontrados mostraram a diversidade de resultados, confirmando a necessidade de mais estudos sobre o tema.

\section{Conclusão}

As práticas de mindfulness e meditação podem funcionar como instrumentos terapêuticos importantes no abrandamento dos sintomas observados no DPOC. Podem ser um complemento valioso à reabilitação pulmonar e à terapia medicamentosa tradicional, já que auxiliam não apenas na diminuição da sensação de dispneia, mas também na diminuição da ansiedade, do estresse e da depressão observados como comorbidade dessa doença.

Apesar disso, poucos trabalhos experimentais sobre o tema foram publicados nos últimos 11 anos, deixando evidente a necessidade de uma pesquisa mais aprofundada

\section{References}

1.World Health Organization. Chronic of respiratory disease. Burden of COPD. [homepage on the Internet]. [2013 January 25]. Disponível em: http:// www.who.int/respiratory/copd/burden/ en/ index.html.

2. Projeto Latino-Americano de Investigação em Obstrução Pulmonar (Platino). ALAT (Associação Latino-Americana de Tórax). Universidade Federal de São Paulo, Escola Paulista de Medicina, São Paulo, SP 2006. Disponível em: http://www. platino-alat.org/docs/livro_platino_pt.pdf.

3. Rycroft CE, Heyes A, Lanza L, et al. Epidemiology of chronic obstructive pulmonary disease: a literature review. Int J Chron Obstruct Pulmon Dis. 2012;7:457-494.

4. GOLD. Guidelines Global Strategy for Diagnosis, Management, and Prevention of COPD [internet]. 2016. Disponível em: http:// www.goldcopd.org/guidelines-global-strategy-for-diagnosis-management.html

5. Gupta D, Agarwal R, Aggarwal AN, et al Guidelines for diagnosis and management of chronic obstructive pulmonary disease: Joint ICS/NCCP (I) recommendations. Lung India. 2013:228267.

6. Hillas G, Perlikos F, Tsiligianni I, et al. Managing comorbidities in COPD. Int J Chron Obstruct Pulmon Dis. 2015;10: 95-109.

7. Panagioti $M$, Scott C, Blakemore A, et al. Overview of the prevalence, impact and management of depression and axiety in chronic obstructive pulmonar disease. Int J Chron Obstruct Pulmon Dis. 2014;9:1289-1309. 


\section{Artigo de revisão}

8. Tselebis A, Pachi A, Ilias I, et al. Strategies to improve anxiety and depression in patients with COPD: a mental health perspective. Neuropsychiatr Dis Treat. 2016;12:297-328.

9. Katon W. Epidemiology and treatment of depression in patients with chronic medical illness. Dialogues Clin Neurosci. 2011;13(1):7-23.

10. Castillo ARGL, Recondo R, Asbahr FR, et al. Transtornos de ansiedade. Rev Bras Psiq. 2000;22:20-23.

11. Braga JEF, Pordeus LC, Silva ATMC, et al. Ansiedade Patológica: bases neurais e avanços na abordagem psicofarmacológica. Revista Brasileira de Ciências da Saúde. 2010;14(2):93-100.

12. Chan R, Giardino N, Larson J. A pilot study: mindfulness meditation intervention in COPD. Int J Chron Obstruct Pulmon Dis. 2015;10:445-454.

13. Godoy R. Ansiedade, depressão e desesperança em paciente com DPOC. Estud. Pesqui. Psicol. 2013;13(3).

14. Norweg A, Collins E. Evidence for cognitive-behavioral strategies improving dyspnea and related distress in COPD. Int J Chron Obstruct Pulmon Dis. 2013;8:439-451.

15. Hyland ME, Halpin DMG, Blake $S$, et al. Preference for different relaxation techniques by COPD patients: comparison between six techniques, Int J Chron Obstruct Pulmon Dis. 2016.

16. Bianco S, Paolo B, Palmierl A. Traditional Meditation, Mindfulness and Psychodynamic Approach: An Integrative Perspective. Front Psychol. 2016;7.

17. Farver-Vestergaard, Jacobsen D, Zachariae R. Efficacy of psychosocial interventions on psychological and physical health outcomes in chronic obstructive pulmonary disease: a systematic review and meta-analysis. Psychother Psychosom. 2015;1:37-50.

18. Taneja DK. Yoga and health. Indian J Community Med. 2014:68-72.
19. Howarth A, Perkins-Porras L, Copland C, et al. Views on a brief mindfulness intervention among patients with long-term illness. BMC Psychol. 2016.

20. Benzo RP. Mindfulness and motivational interviewing: two candidate methods for promoting self-management. Chron Respir Dis. 2015:175-182.

21. Mularski RA, Munjas BA, Lorenz KA, et al. Randomized controlled trial of mindfulness-based therapy for dyspnea in chronic obstructive lung disease. J Altern Complement Med. 2009:1083-1090.

22. Harrison SL, Lee A, Goldstein RS, et al. Perspectives of healthcare professionals and patients on the application of mindfulness in individuals with chronic obstructive pulmonary disease. Patient Educ Couns. 2016.

23. Chan RR, Lehto RH. The Experience of Learning Meditation and Mind/Body Practices in the COPD Population. Explore (NY). 2016:171-179.

24. Fulambarker A, Farooki B, Kheir F, et al. Effect of yoga in chronic obstructive pulmonary disease. Am J Ther. 2012:96-100.

25. Yeh GY, Roberts DH, Wayne PM, et al. Tai chi exercise for patients with chronic obstructive pulmonary disease: a pilot study. Respir Care. 2010:1475-1485.

26. Ranjita R, Hankey A, Negendra HR, et al. Yoga-based pulmonary rehabilitation for the management of dyspnea in coal miners with chronic obstructive pulmonary disease: A randomized controlled trial. J Ayurveda Integr Med. 2016:158-166.

27. Selman L, Mcdermott K, Donesky D, et al. Appropriateness and acceptability of a Tele-Yoga intervention for people with heart failure and chronic obstructive pulmonary disease: qualitative findings from a controlled pilot study. BMC Complement Altern Med. 2015:15-21. 